BNL-112682-2016-JA

\title{
Advancing Reversible Shape Memory by Tuning the Polymer Network Architecture
}

Qiaoxi Li, Jing Zhou, Mohammad Vatankhah-Varnoosfaderani,
Dmytro Nykypanchuk, Oleg Gang and Sergei S. Sheiko

Submitted to Nanostar $U$

February 2016

Center for Functional Nanomaterials

Brookhaven National Laboratory

\author{
U.S. Department of Energy \\ USDOE Office of Science (SC), \\ Basic Energy Sciences (SC-22)
}




\section{DISCLAIMER}

This report was prepared as an account of work sponsored by an agency of the United States Government. Neither the United States Government nor any agency thereof, nor any of their employees, nor any of their contractors, subcontractors, or their employees, makes any warranty, express or implied, or assumes any legal liability or responsibility for the accuracy, completeness, or any third party's use or the results of such use of any information, apparatus, product, or process disclosed, or represents that its use would not infringe privately owned rights. Reference herein to any specific commercial product, process, or service by trade name, trademark, manufacturer, or otherwise, does not necessarily constitute or imply its endorsement, recommendation, or favoring by the United States Government or any agency thereof or its contractors or subcontractors. The views and opinions of authors expressed herein do not necessarily state or reflect those of the United States Government or any agency thereof. 


\title{
Advancing Reversible Shape Memory by Tuning the
}

\section{Polymer Network Architecture}

Qiaoxi Li, ${ }^{1}$ Jing Zhou, ${ }^{1}$ Mohammad Vatankhah-Varnoosfaderani, ${ }^{1}$ Dmytro Nykypanchuk, ${ }^{2}$ Oleg Gang, ${ }^{2}$ Sergei S. Sheiko ${ }^{1 *}$

${ }^{1}$ Department of Chemistry, University of North Carolina at Chapel Hill, NC 27599, United States

${ }^{2}$ Center for Functional Nanomaterials, Brookhaven National Laboratory, Upton NY 11973

\begin{abstract}
Due to counteraction of a chemical network and a crystalline scaffold, semi-crystalline polymer networks exhibit a peculiar behavior - reversible shape memory (RSM), which occurs naturally without applying any external force and particular structural design. There are three RSM properties: (i) range of reversible strain, (ii) rate of strain recovery, and (iii) decay of reversibility with time, which can be improved by tuning the architecture of polymer network. Different types of poly(octylene adipate) networks were synthesized allowing for control of crosslink density and network topology, including randomly cross-linked network by free-radical polymerization, thiol-ene clicked network with enhanced mesh uniformity, and loose network with deliberately incorporated dangling chains. It is shown that the RSM properties are controlled by average crosslink density and crystal size, whereas topology of a network greatly affects its extensibility. We have achieved $80 \%$ maximum reversible range, $15 \%$ minimal decrease in reversibility, and fast strain recovery rate up to $0.05 \mathrm{~K}^{-1}$, i.e. ca. $5 \%$ per $10 \mathrm{~s}$ at a cooling rate of $5 \mathrm{~K} / \mathrm{min}$.
\end{abstract}


for Table of Contents use only

\section{Advancing Reversible Shape Memory by Tuning the}

\section{Polymer Network Architecture}

Qiaoxi Li, ${ }^{1}$ Jing Zhou, ${ }^{1}$ Mohammad Vatankhah-Varnoosfaderani, ${ }^{1}$ Dmytro Nykypanchuk, ${ }^{2}$ Oleg Gang, ${ }^{2}$ Sergei S. Sheiko ${ }^{1 *}$

${ }^{1}$ Department of Chemistry, University of North Carolina at Chapel Hill, NC 27599, United States

${ }^{2}$ Center for Functional Nanomaterials, Brookhaven National Laboratory, Upton NY 11973
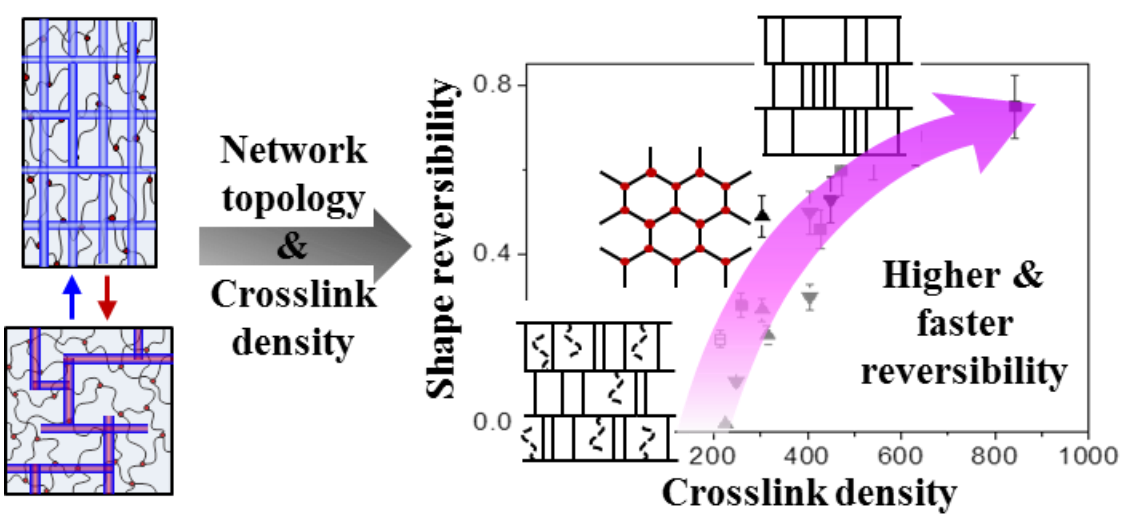


\section{INTRODUCTION}

Soft materials that are capable of reversible shape alterations are vital for the development of actuators, artificial muscles, and haptic devices ${ }^{[1-4]}$. Of these materials, shape memory polymers (SMPs) show unique potential to encode complex and re-programmable shapes ${ }^{[5-7]}$. However, this advantage is offset by the intrinsic irreversibility of conventional shape-memory transformations. The irreversibility issue can be resolved by liquid-crystal elastomers with synthetically encoded alignment of mesogens ${ }^{[8-12]}$, mechanically assisted SMPs ${ }^{[13-15]}$ and composites built of oppositely strained elements ${ }^{[16]}$. Recently, reversible shape memory (RSM) has been realized in conventional semi-crystalline polymer networks without applying any external force and synthetic programming ${ }^{[17,18]}$. As shown in Figure 1, during partial melting of a programmed shape, relaxation of a strained chemical network is reversed by re-crystallization of the crystalline scaffold upon cooling. Even though network relaxation is thermodynamically favored, crystallization of cross-linked strands follows kinetically preferred pathways imposed by the scaffold of the programmed shape.

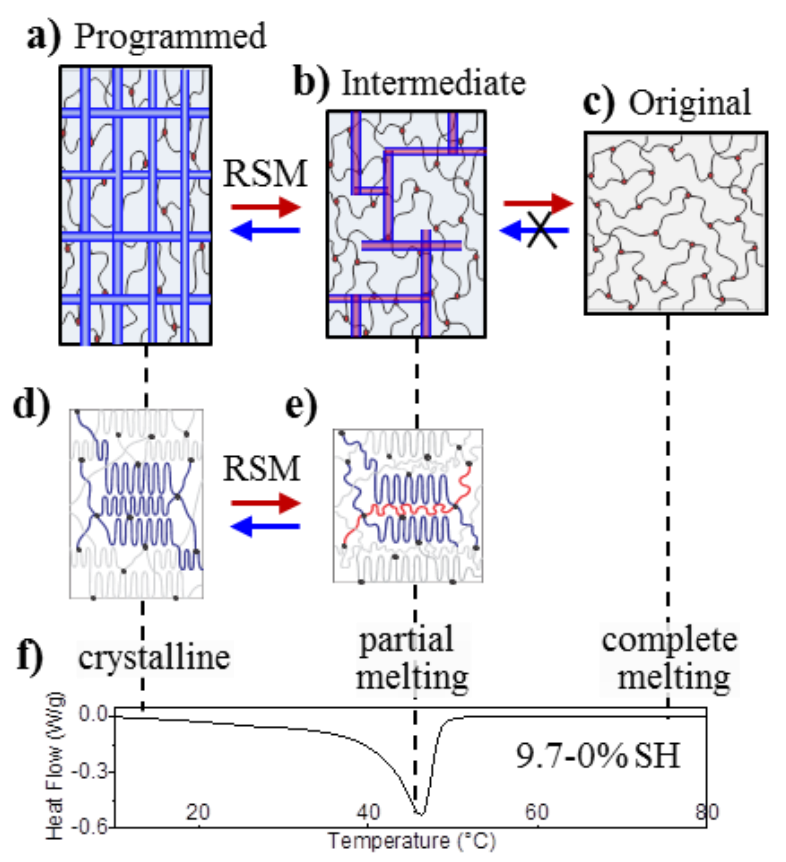


Figure 1. Macroscopic shape and mesoscopic crystalline scaffold transformations. a) A programmed shape was fixed by a percolated crystalline scaffold (blue bars). b) Upon partial melting, the remaining crystals serve as a template for self-seeding crystallization. Upon cooling of the intermediate shape, the recrystallization process causes reversible shape-shifting towards the programmed shape, hence reversible shape memory (RSM). c) On complete melting, the original equilibrium shape is recovered and shape memory becomes irreversible. d) and e) show molecular interpretation of self-seeding crystallization. On partial melting e), the molten chains (shown in red) are constrained by remaining crystallites and chemical crosslinks. Therefore, on cooling d), recrystallization of these chains replicates the original scaffold restoring the programmed shape. f) Heat flow measured by Differential Scanning Calorimetry (DSC) shows different stages of the melting process of one of the studies polymers (9.7-0\% SH, Table 1).

This RSM mechanism led to several practical applications, including robotic gripper ${ }^{[18]}$, switchable micro-patterned surface ${ }^{[19]}$, dynamic optical grating ${ }^{[20]}$, isothermal programming of triple shapes ${ }^{[21]}$. The goal of the present study is to explore structure-property correlations of semi-crystalline octylene-adipate polymer networks and expand the RSM limits with respect to (i) range of reversible strain, (ii) rate of reversed strain recovery, and (iii) decay of the reversibility with time. For this purpose, we have prepared polymer networks with different topologies, including irregular network, clicked regular network, and loose network with dangles. Additionally, using oligomers with different degree of oligomerizations, we control crosslinking density in a broad range of ca. $50-1000 \mathrm{~mol} / \mathrm{m}^{3}$. While controlling the crosslink density, we ensure that the densest network has sufficient amount of crystals to fix a programmed shape and the loosest network has enough elasticity to recover its original state. In this paper, we show that the RSM properties display significant shifts with increasing crosslink density, whereas network topology does not cause any specific impact on RSM. The crosslink density has dual effect on the network properties. It controls both elasticity of the chemical network, which provides a restoring force towards the original shape, and dimensions of the crystalline scaffold, which 
secures memory of the programmed shape. By managing the interplay between the chemical and crystalline networks, we achieved nearly $80 \%$ recovery of the programmed strain at a high rate of $0.05 \mathrm{~K}^{-1}$ and less than $15 \%$ decay of reversibility after isothermal annealing for 2 hours.

\section{EXPERIMENTAL SECTION}

Synthesis of octylene-adipate (OA) oligomers. All chemicals were used as purchased from Sigma-Aldrich. In a $100 \mathrm{~mL}$ dried two-way round bottom flask that connected to a condenser, 1,8-octane diol (4.0 g, $2.74 \mathrm{mmol})$ was dissolved in $40 \mathrm{~mL}$ anhydrous $\mathrm{CH}_{2} \mathrm{Cl}_{2}$ under mild heating condition $\left(35^{\circ} \mathrm{C}\right)$. Triethylamine $(0.5 \mathrm{~g}, 5.04 \mathrm{mmol})$ was added into the mixture. Adipoyl chloride (4.6 g, $2.52 \mathrm{mmol})$ was injected into the flask using a syringe pump at a rate of $1 \mathrm{~mL} / \mathrm{hr}$ while the mixture was stirring. After injection, the reaction was left for an additional hour. The oligomerization was terminated by filtering the salt triethylamine hydrochloride and then extracting excess of solvent followed by precipitation with methanol in ice bath. Precipitates were filtered and then dissolved in $40 \mathrm{~mL}$ dichloromethane and dried by adding anhydrous magnesium sulfate. The product solution was filtered and solvent was removed by rotary evaporator followed by drying under vacuum at room temperature overnight. Based on theory of step growth polymerization, oligomers have the same end-group of the excess monomer. In this study, we used excess amount of diol monomer. The molecular weight of oligomers can be controlled by the ratio of two monomers (1,8-octane diol and adipoyl chloride). Different monomers ratios and the corresponding degree of oligomerization $n$ (including theoretical $n$ predicted by Carothers equation [22] and experimental $n$ measured from 1H-NMR) are summarized in Table S1 in Supporting Information. 1H-NMR $\left(400 \mathrm{MHz}, \mathrm{CDCl}_{3}\right): \delta(\mathrm{ppm})=$ 4.07 (t, $4 n \mathrm{H}), 3.66(\mathrm{t}, 4 \mathrm{H}), 2.34$ (t, $4 n \mathrm{H})$, [1.68 \& 1.63] (broad, -OCOCH $\left.2_{2}-\left(\mathrm{CH}_{2}\right)_{2}-\right), 1.34$ (broad, 
$\left.-\mathrm{COOCH}_{2}-\left(\mathrm{CH}_{2}\right)_{6}{ }^{-}\right)$. Degree of oligomerization $n$ was calculated as the ratio of area of peaks in $\delta$ $=4.07 \mathrm{ppm} / \delta=3.66 \mathrm{ppm}$. See Supporting Information for more details (Figure S1).

End functionalization of OA oligomers. Mono-end functionalized oligomers were synthesized by the reaction of dissolved OA oligomers $(2.0 \mathrm{~g}, 0.68 \mathrm{mmol})$ in $20 \mathrm{~mL}$ anhydrous $\mathrm{CH}_{2} \mathrm{Cl}_{2}$ with slowly added 2-isocyanatoethyl methacrylate $(0.1 \mathrm{~g}, 0.68 \mathrm{mmol})$ in the presence of dibutyltin dilaurate (DBTDL) $(0.01 \mathrm{~g}, 0.016 \mathrm{mmol})$ as a catalyst in a $100 \mathrm{~mL}$ dried round bottom flask. After injection, the reaction was left overnight. The reaction was terminated by extracting excess of solvent and precipitated with methanol in ice bath. Precipitates were filtered and dried under vacuum at room temperature overnight to extract any remaining solvent. $1 \mathrm{H}-\mathrm{NMR}(400 \mathrm{MHz}$, $\left.\mathrm{CDCl}_{3}\right): \delta(\mathrm{ppm})=6.15(\mathrm{~s}, 4 \mathrm{H}), 5.62(\mathrm{~s}, 4 \mathrm{H}), 4.25(\mathrm{t}, 8 \mathrm{H}), 4.07(\mathrm{t}, 12 n \mathrm{H}), 3.66(\mathrm{q}, 4 \mathrm{H}), 3.52(\mathrm{q}$, 8H), $2.34(\mathrm{t}, 12 n \mathrm{H}), 1.97(\mathrm{~s}, 12 \mathrm{H})$, [1.68 \&1.63] (broad, -OCOCH$\left.{ }_{2}-\left(\mathrm{CH}_{2}\right)_{2}-\right)$, 1.34 (broad, $\left.\mathrm{COOCH}_{2}-\left(\mathrm{CH}_{2}\right)_{6^{-}}\right)$. The ratio of mono-end capped chains (dangles) and both-end capped chains has been calculated as the ratio of $\delta=3.52 \mathrm{ppm} / \delta=3.66 \mathrm{ppm}$, which is equal to $2 / 1$. Therefore, the mol\% of dangles is determined as $67 \mathrm{~mol} \%$. See Figure S2 in Supporting Information.

To functionalize both end of OA oligomers with methacrylate group, excess amount of 2isocyanatoethyl methacrylate $(0.3 \mathrm{~g}, 1.7 \mathrm{mmol})$ was added into OA oligomers $(2.0 \mathrm{~g}, 0.68 \mathrm{mmol})$ in $20 \mathrm{~mL} \mathrm{CH}_{2} \mathrm{Cl}_{2}$. 1H-NMR (400MHz, $\left.\mathrm{CDCl}_{3}\right): \delta(\mathrm{ppm})=6.15(\mathrm{~s}, 2 \mathrm{H}), 5.62(\mathrm{~s}, 2 \mathrm{H}), 4.25(\mathrm{t}, 4 \mathrm{H})$, $4.07(\mathrm{t}, 4 n \mathrm{H}), 3.52(\mathrm{q}, 4 \mathrm{H}), 2.34(\mathrm{t}, 4 n \mathrm{H}), 1.97(\mathrm{~s}, 6 \mathrm{H})$, [1.68 \& 1.63] (broad, $-\mathrm{OCOCH}_{2}-\left(\mathrm{CH}_{2}\right)_{2}-$ ), 1.34 (broad, $\left.-\mathrm{COOCH}_{2}-\left(\mathrm{CH}_{2}\right)_{6}{ }^{-}\right)$. Disappearance of $\delta=3.66 \mathrm{ppm}$ and appearance of capped methacrylate peaks proved full conversion of hydroxyl groups into methacrylate groups at chain ends. See Figure S3 in Supporting Information. 
Networks fabrication by photo-induced free-radical polymerization. A series of poly(octylene adipate) (POA) networks with different crosslink densities was prepared by UVcrosslinking of OA oligomers with different degrees of oligomerization $n$. In a dry vial, bifunctionalized $\mathrm{OA}$ oligomers were dissolved in $\mathrm{CHCl}_{3}$ at $50 \mathrm{wt} \%$ ratio. 1 wt\% of 2, 2diethoxyacetophenone (DEAP) was added into the mixture and vortex was used to thoroughly mix all components. The mixture was transferred into a syringe and injected into a PTFE mold covered with a quartz slide on top to prevent solvent evaporation. The filled mold was exposed to UV-365 nm for 5 minutes under nitrogen blanket to form fully cured network. The fabricated network was then taken out and dried in vacuum oven at $80^{\circ} \mathrm{C}$ overnight. Due to uncontrolled radical activation, the resulting network has a dense and irregular topology.

In addition, dangling chain ends were introduced into the above network by using mixture of bi-functionalized oligomers and mono-functionalized oligomers. These networks were fabricated in different mole ratios of bi-functionalized oligomers to mono-functionalized oligomers: 100/0 (0\% dangles), 60/40 (40\% dangles), 33/67 (67\% dangles). All molecular parameters of the irregular networks prepared by free-radical polymerization are summarized in Table 1.

Networks fabrication by thiol-ene click reaction. Stoichiometric tri-thiol cross-linker, trimethylolpropane tris (3-mercaptopropionate) was mixed with bi-functionalized OA oligomers at different mole ratios of -SH/-methacrylate: $1 / 1(100 \% \mathrm{SH}), 0.5 / 1(50 \% \mathrm{SH})$. UV curing was applied in the same way. Note that reactivity of the click reaction decreases with oligomer size due to viscosity enhancement, which increases the contribution of free-radical polymerization. As will show later in Figure 2c, for a short oligomer $n=6.7$, the crosslink density of $6.7-100 \%$ SH sample nearly drops to $1 / 3$ of $6.7-0 \%$ SH sample, while the longer oligomer $n=23.1$ exhibits 
a much less decrease in crosslink density due to kinetic domination of the free-radical polymerization. The molecular parameters of the "clicked" networks are summarized in Table 1.

The sol-gel fraction of all prepared networks has been measured using Soxhlet extractor in $\mathrm{CHCl}_{3}$ at $65^{\circ} \mathrm{C}$ overnight. All samples show gel fraction $>95 \%$.

Differential Scanning Calorimetry. A heat/cool/heat cycle with a ramp rate of $5 \mathrm{~K} / \mathrm{min}$ from 0 ${ }^{\circ} \mathrm{C}$ to $80{ }^{\circ} \mathrm{C}$ was used for all samples by DSC 220 from Seiko Instruments, Inc. Second heating was used to determine the melting transition, while cooling was used to characterize the crystallization transition. The crystallinity was calculated by the ratio of the integration of heating or cooling transition to the heat of fusion of octylene-adipate polymers, which is close to $150 \mathrm{~J} / \mathrm{g}^{[23]}$.

Dynamic Mechanical Analysis. RSA-G2 dynamic mechanical analyzer from TA Instruments was used for mechanical tests. Storage modulus was measured in oscillation mode at $1 \mathrm{~Hz}, 0.1 \%$ strain. Extensibility was measured at $80^{\circ} \mathrm{C}$ with a linear elongation rate of $0.5 \mathrm{~mm} / \mathrm{s}$.

In a conventional shape-memory test, a targeted programming strain of $\varepsilon_{p}=20 \%$ is applied to a sample with original shape $\varepsilon_{o}$ at $80{ }^{\circ} \mathrm{C}$ (typically, $\varepsilon_{o}=0$ ). Then the sample is cooled $\left(5 \mathrm{~K} / \mathrm{min}\right.$ ) to $0^{\circ} \mathrm{C}$, while maintaining the same programming strain $\varepsilon_{p}$. After releasing the load and annealing for $1 \mathrm{~min}$ at $0{ }^{\circ} \mathrm{C}$, strain relaxes from $\varepsilon_{p}$ to a fixed strain $\varepsilon_{f}$ - programmed shape. Upon heating the sample to $80^{\circ} \mathrm{C}(5 \mathrm{~K} / \mathrm{min})$, the shape recovers strain to $\varepsilon_{r}$, which is irreversible. To quantify shape fixation and recovery, we use the following parameters:

$$
\text { Fixation ratio }=\frac{\varepsilon_{f}}{\varepsilon_{p}}
$$




$$
\text { Recovery ratio }=\frac{\varepsilon_{r}-\varepsilon_{f}}{\varepsilon_{o}-\varepsilon_{f}}
$$

All samples in this study have fixation ratio and recovery ratio $>95 \%$, therefore we simplify the notations: the fixed shape is the same as programmed shape $\left(\varepsilon_{f}=\varepsilon_{p}\right)$, whereas the irreversibly recovered shape is the same as original shape $\left(\varepsilon_{r}=\varepsilon_{o}\right)$, which is typically zero $\left(\varepsilon_{o}=0\right)$.

To conduct RSM, a sample of a programmed shape $\left(\varepsilon_{p}\right)$, was heated to a partial melting temperature $T_{\text {partial }}$, which was close to the melting peak $T_{m}$ on DSC curves. Upon heating (5 $\mathrm{K} / \mathrm{min}$ ), the programmed shape relaxed to an intermediate state characterized by strain $\varepsilon_{i}$. After short annealing time $\Delta t$ at $T_{\text {partial }}$ (no less than $100 \mathrm{~s}$ to assure uniform temperature distribution), subsequent cooling induced reverse shape transformation towards its programmed shape resulting in a strain increase from $\varepsilon_{i}$ to $\varepsilon_{r e v}$ - reversed strain. To quantify RSM, we introduce the following parameter:

$$
\text { Reversibility }=\frac{\varepsilon_{r e v}-\varepsilon_{i}}{\varepsilon_{p}}
$$

Atomic Force Microscopy. Oligomers were dissolved in $\mathrm{CHCl}_{3}(1 \mathrm{wt} \%)$ followed by addition of DEAP $(0.01 \mathrm{wt} \%)$. The solution was spin-coated onto a mica surface at $1500 \mathrm{rpm}$ for $1 \mathrm{~min}$, then placed on a hot plate $\left(80^{\circ} \mathrm{C}\right)$ to keep oligomers in a melt state. Then the film was cured by UV-365 nm for 5 minutes under nitrogen blanket. AFM images were taken using NanoScope V controller in PeakForce QNM mode (Bruker).

Small Angle X-ray Scattering (SAXS). Measurements were conducted at Brookhaven National Lab, Center for Functional Nanomaterials, using a Bruker Nanostar U instrument equipped with Vantec 2000 area detectors and Copper rotating anode as a source. Scattering patterns from the samples were measured at $0{ }^{\circ} \mathrm{C}$ and collection time was set as $30 \mathrm{~s}$ for all samples. A 
polynominal decay function with Savitzky-Golay filter was used for baseline fitting. After baseline subtraction, the peaks were fitted using Lorentz function to determine the full-width-athalf-maximum (FWHM). All mechanical and morphological characteristics of the POA networks are summarized in Table 1.

Table 1. Molecular structure and physical properties of poly(octylene adipate) networks.

\begin{tabular}{|c|c|c|c|c|c|c|c|c|c|c|c|c|c|c|}
\hline$n^{a}$ & mol\% & mol\% & $E^{d}$ & $T_{m}{ }^{e}$ & $T_{c}{ }^{e}$ & $1 H_{m}$ & $T_{\text {partial }}^{\max }{ }^{f}$ & $v^{g}$ & $\lambda_{\max }{ }^{h}$ & $L_{S A X S}{ }^{i}$ & $d L_{S A X S}^{j}$ & $L_{A F M}{ }^{k}$ & $M_{0}{ }^{l}$ & $M_{x}{ }^{m}$ \\
\hline & & & $\mathrm{MPa}$ & ${ }^{\circ} \mathrm{C}$ & ${ }^{\circ} \mathrm{C}$ & $\mathrm{J} / \mathrm{g}$ & ${ }^{\circ} \mathrm{C}$ & $\mathrm{mol} / \mathrm{m}^{3}$ & & $\mathrm{~nm}$ & (FWHM) & $\mathrm{nm}$ & $\mathrm{kg} / \mathrm{mol}$ & $\mathrm{kg} / \mathrm{mol}$ \\
\hline 2.7 & 0 & 0 & 7.5 & 27 & -2 & 36 & 26 & 842 & 1.3 & 13.4 & 0.027 & $\mathrm{n} / \mathrm{a}$ & 1.2 & .4 \\
\hline 6.7 & 0 & 0 & 5.6 & 39 & 13 & 42 & 39 & 630 & 1.6 & 14.0 & 0.024 & $\mathrm{n} / \mathrm{a}$ & 2.3 & .8 \\
\hline 6.7 & 50 & 0 & 4.0 & 46 & 24 & 51 & 44 & 450 & 1.7 & 16.1 & 0.023 & $\mathrm{n} / \mathrm{a}$ & 2.4 & 1.6 \\
\hline 6.7 & 100 & 0 & 2.7 & 48 & 28 & 50 & 46 & 304 & 1.8 & 16.4 & 0.022 & $\mathrm{n} / \mathrm{a}$ & 2.5 & 2.5 \\
\hline 9.7 & 0 & 0 & 4.8 & 47 & 25 & 53 & 45 & 540 & 1.7 & 15.0 & 0.022 & 18 & 3.1 & 1.0 \\
\hline 9.7 & 50 & 0 & 3.6 & 50 & 30 & 54 & 49 & 405 & 2.0 & 16.5 & 0.022 & $\mathrm{n} / \mathrm{a}$ & 3.2 & 2.1 \\
\hline 9.7 & 100 & 0 & 2.7 & 56 & 32 & 60 & 50 & 304 & 2.5 & 16.6 & 0.021 & $\mathrm{n} / \mathrm{a}$ & 3.3 & 3.3 \\
\hline 14.7 & 0 & 0 & 4.2 & 54 & 30 & 64 & 51 & 472 & 1.7 & 15.7 & 0.024 & 20 & 4.4 & 1.5 \\
\hline 14.7 & 50 & 0 & 3.6 & 55 & 31 & 65 & 53 & 405 & 1.7 & 16.8 & 0.022 & $\mathrm{n} / \mathrm{a}$ & 4.6 & 3.0 \\
\hline 14.7 & 100 & 0 & 2.8 & 56 & 34 & 65 & 53 & 315 & 2.7 & 17.5 & 0.021 & $\mathrm{n} / \mathrm{a}$ & 4.7 & 4.7 \\
\hline 23.1 & 0 & 0 & 2.3 & 60 & 37 & 73 & 58 & 259 & 1.8 & 18.5 & 0.022 & 22 & 6.7 & 2.2 \\
\hline 23.1 & 50 & 0 & 2.2 & 61 & 41 & 73 & 59 & 248 & 3.3 & 18.2 & 0.021 & $\mathrm{n} / \mathrm{a}$ & 6.8 & 4.6 \\
\hline 23.1 & 100 & 0 & 2.0 & 62 & 41 & 75 & $\mathrm{n} / \mathrm{a}$ & 225 & 4.8 & 18.5 & 0.021 & $\mathrm{n} / \mathrm{a}$ & 7.0 & 7.0 \\
\hline 15.1 & 0 & 0 & 3.8 & 54 & 32 & 64 & 51 & 428 & 1.6 & 16.1 & 0.023 & $\mathrm{n} / \mathrm{a}$ & 4.6 & 1.5 \\
\hline 15.1 & 0 & 40 & 1.9 & 59 & 38 & 72 & 52 & 214 & 2.5 & 17.3 & 0.021 & $\mathrm{n} / \mathrm{a}$ & 4.6 & 2.5 \\
\hline 15.1 & 0 & 67 & 0.6 & 63 & 43 & 82 & $\mathrm{n} / \mathrm{a}$ & 68 & 4.0 & 18.0 & 0.020 & $\mathrm{n} / \mathrm{a}$ & 4.6 & 4.6 \\
\hline
\end{tabular}

${ }^{a}$ Degree of polymerization of functionalized OA oligomers (Figure 2a). ${ }^{b}$ Molar fraction of trimethylolpropane tris (3-mercaptopropionate) (Figure 2a). ${ }^{c}$ Molar fraction of monofunctional OA oligomers (Figure 2a). ${ }^{d}$ Storage modulus measured by DMA using oscillation mode at $1 \mathrm{~Hz}, \varepsilon=0.001, T=80{ }^{\circ} \mathrm{C}$. ${ }^{e}$ Melting peak, crystallization peak, and melting enthalpy (integration of melting peak) measured by DSC at $5 \mathrm{~K} / \mathrm{min} .{ }^{f}$ The optimum partial melting temperature at which the maximum reversibility was obtained. Samples $23.1-100 \%$ SH and $15.1-67 \%$ dangles showed no reversibility. ${ }^{g}$ Crosslink density $v=G_{80^{\circ}} \mathrm{C} R T=E_{80^{\circ} \mathrm{C}} 3 R T, G_{80^{\circ} \mathrm{C}}$ is shear modulus. ${ }^{h}$ Extensibility $\lambda_{\max }=L_{\max } / L_{0} . L_{\max }$ is length at break, $L_{0}$ is original length. ${ }^{i}$ Average long period of lamellae $L_{S A X S}=2 \pi / q_{\max } . q_{\max }$ is peak position of SAXS profile. ${ }^{j}$ Distribution of lamellae long period determined by the full width at half maximum of SAXS peak. ${ }^{k}$ Average spacing of lamellae on AFM height profile. ${ }^{l}$ Molecular weight of functionalized oligomers based on chemical structure. ${ }^{m}$ Average molecular weight of network strand depends on the network 
topology as $M_{0} / 3$ for $0 \% \mathrm{SH}, 2 M_{0} / 3$ for $50 \% \mathrm{SH}, M_{0}$ for $100 \% \mathrm{SH},\left(M_{0} / 3\right) /(1-$ mol $\%$ dangles $)$ for networks with dangled chains. All samples have gel fraction $>95 \%$, fixation ratio and recovery ratio $>95 \%$.

\section{RESULTS AND DISCUSSION}

Different formulations of dimethacrylates, monomethacrylates, tri-thiol crosslinkers (Figure 2a) were utilized to prepare three distinct network topologies (Figure 2b). In all three synthetic protocols, we varied degree of oligomerization $n$ of the oligomers (from 2.7 to 23.1) to cover a wide range of crosslink density (Table 1). We did not pursue higher crosslink densities as they imped the crystallization process and disallow shape fixation. We also did not study lower crosslink densities since this would lead to reduction of the restoring force and disable shape memory. Photo-induced free-radical polymerization of bi-functional oligomers (1) resulted in an irregular network I (Figure 2b) and high crosslink density (Figure 2c). For example, sample 2.7$0 \%$ SH ( $n=2.7$ of $\mathbf{1}$ and $0 \%$ of $\mathbf{3})$ shows the highest crosslink density $\left(842 \mathrm{~mol} / \mathrm{m}^{3}\right)$ and lowest values in $T_{c}\left(-2{ }^{\circ} \mathrm{C}\right)$ and $\Delta H_{m}(36 \mathrm{~J} / \mathrm{g})$, which indicates constrained crystallization within a dense network yielding lower crystallinity and smaller crystallites. Additionally (network II), we mixed bi- and mono-functional oligomers $(1+2)$ to introduce dangling chains, which do not support external stress and act as diluents decreasing the crosslinking density. Dangles also enhance crystallization, which is evidenced by rise of $T_{c}$ from $32{ }^{\circ} \mathrm{C}$ to $43{ }^{\circ} \mathrm{C}$ and $\Delta H_{m}$ from 64 $\mathrm{J} / \mathrm{g}$ to $82 \mathrm{~J} / \mathrm{g}$ with increasing mol\% of dangles (Table 1). To enable better control of the network topology, we applied thiol-ene click chemistry $(\mathbf{1}+\mathbf{3})$, which allows much faster reaction rate between methacrylate-SH than methacrylate self-polymerization ${ }^{[24,25]}$. This synthetic approach yields networks III with better mesh uniformity, which resulted in lower modulus (Figure 2c) and significant enhancement in extensibility (Figure 1d). 
a) Chemical structures

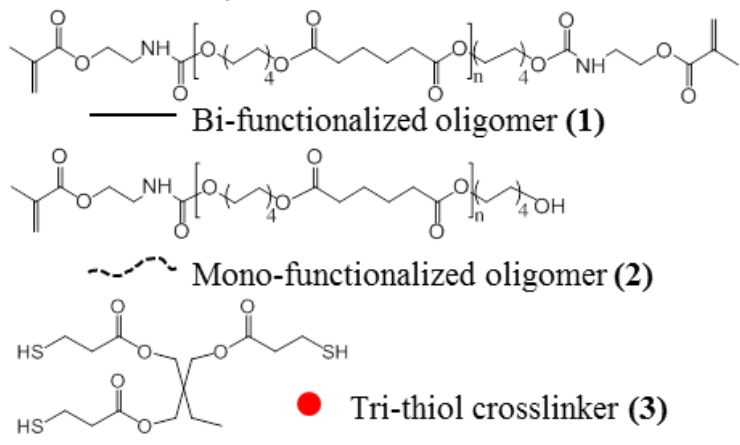

b) Network topologies
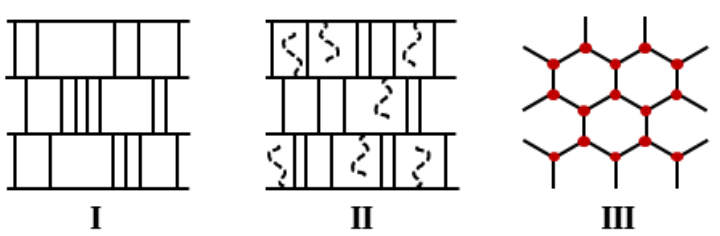

c) Modulus

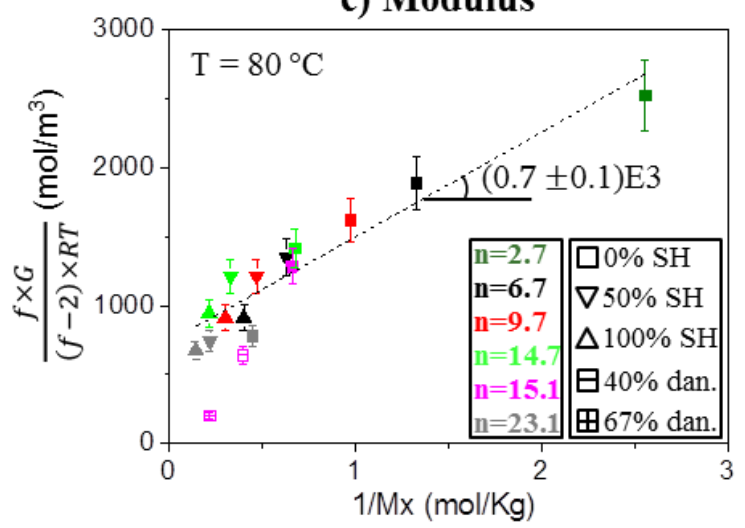

d) Extensibility

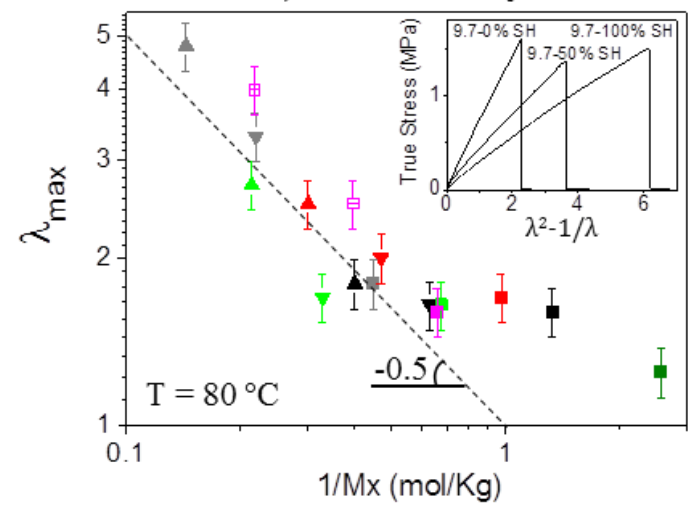

Figure 2. Network design and control parameters. a) $\mathrm{Bi}$ - (1) and mono- (2) endfunctionalized octylene-adipate (OA) oligomers with an average degree of oligomerization $n$ varied from 2.7 to 23.1 (Table 1), and (3) tri-thiol cross-linkers. b) Irregular topology prepared by free-radical polymerization of (1); dangling chains incorporated by free-radical copolymerization of (1) and (2); uniform topology prepared by click reaction of (1) and (3). c) The temperature-normalized shear modulus $\frac{f}{f-2} \frac{G}{R T}$ increases upon decreasing the average molar mass of network strand $M_{x}$. This modulus corresponds to molar crosslink density $v$ (number of moles of mechanically active network strands per unit volume), where $f=3$ - branching functionality of network junctions and $G$ - shear modulus measured at $T=80^{\circ} \mathrm{C}$. The dashed line is a linear fit consistent with the classical crossover equation for an entangled polymer network $v \cong v_{e}+\frac{\rho}{M_{x}}$ ${ }^{[26]}$, excluding samples with dangles that are described by a different model with 'loose-end' correction ${ }^{[27]}$ ), where $v_{e}$ is the density of chain entanglements. d) Extensibility $\lambda_{\max }=$ $L_{\text {max }} / L_{0}$ measured at $T=80{ }^{\circ} \mathrm{C}$ decreases with $1 / M_{x}$. Samples with $(\mathbf{1 + 3})$ topology follow a linear trend with a slope of -0.5 , which is consistent with $\lambda_{\max } \sim \sqrt{M_{x}}$, theoretically expected for ideal networks. The inset shows true stress-true strain curve until break. 
Shear modulus $G$ of polymer networks was measure in a melt state at $T=80{ }^{\circ} \mathrm{C}$. Figure 2c depicts the average molar crosslinking density $v \cong \frac{f}{f-2} \frac{G}{R T}$ versus the number-average molar mass of the strands $M_{x}$ for different network topologies with $f=3$ (functionality of the crosslink junctions). The slope $0.7 \cdot 10^{3} \mathrm{~kg} / \mathrm{m}^{3}$ of the linear fit (dashed line) is in good agreement with the polymer mass density $\rho \cong 10^{3} \mathrm{~kg} / \mathrm{m}^{3}$, whereas the intercept $v_{e}=200 \mathrm{~mol} / \mathrm{m}^{3}$ and the corresponding shear modulus of $G_{e} \cong \frac{f-2}{f} v_{e} R T \cong 1.7 \cdot 10^{5} \mathrm{~Pa}$ are consistent with the respective characteristics of a typical entangled network $\left(\nu_{e} \sim 100 \mathrm{~mol} / \mathrm{m}^{3}\right.$ and $\left.G_{e} \sim 10^{5} \mathrm{~Pa}\right)$ ${ }^{[28]}$. These agreements, along with the characteristic slope in Figure 2d, indicate good synthetic control of the network topology and the crosslink density.

Network topology exhibits significant effect on samples' extensibility $\left(\lambda_{\max }\right)$, which was measured in a melt state at $T=80^{\circ} \mathrm{C}$. As seen in Figure $2 \mathrm{~d}$, the irregular dense networks (I) demonstrate poor extensibility $\left(\lambda_{\max }<2\right)$ due to highly non-uniform stress distribution over the network strands. In contrast, the more uniform networks (III) and the network with dangling chains (II) allow extensions up to 5-times. As seen in the inset in Figure 1d, three samples with the same $n=9.7$ exhibit almost the same stress-at-break ( 1.5 MPa), while the strain-at-break increases up to three times as SH\% increases. These loose networks show excellent shape memory behavior ( 100\% shape fixity and recovery) at high programming strains exceeding $100 \%$. However, as discussed below, the shape reversibility decreases upon lowering crosslink density. In fact, one of the most irregular and dense networks studied in this paper $(2.7-0 \% \mathrm{SH})$ exhibits the best reversibility (ca. $80 \%$ of the programmed strain), while possessing the highest modulus and worst extensibility. 
a)
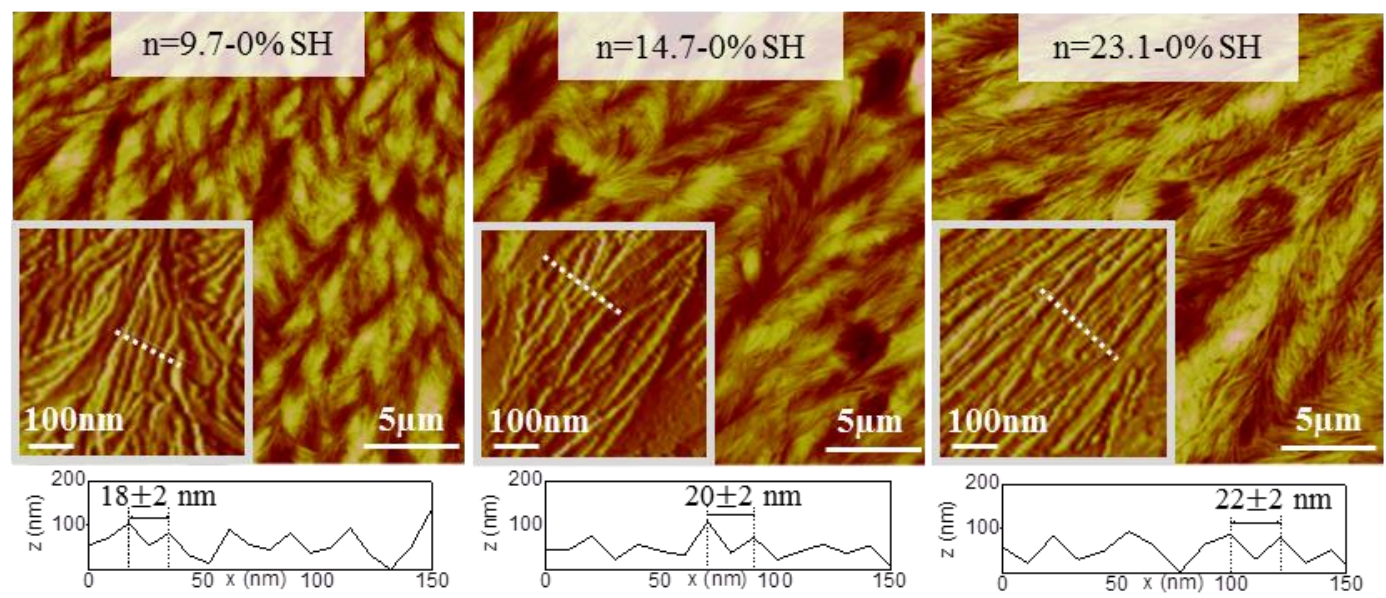

b)
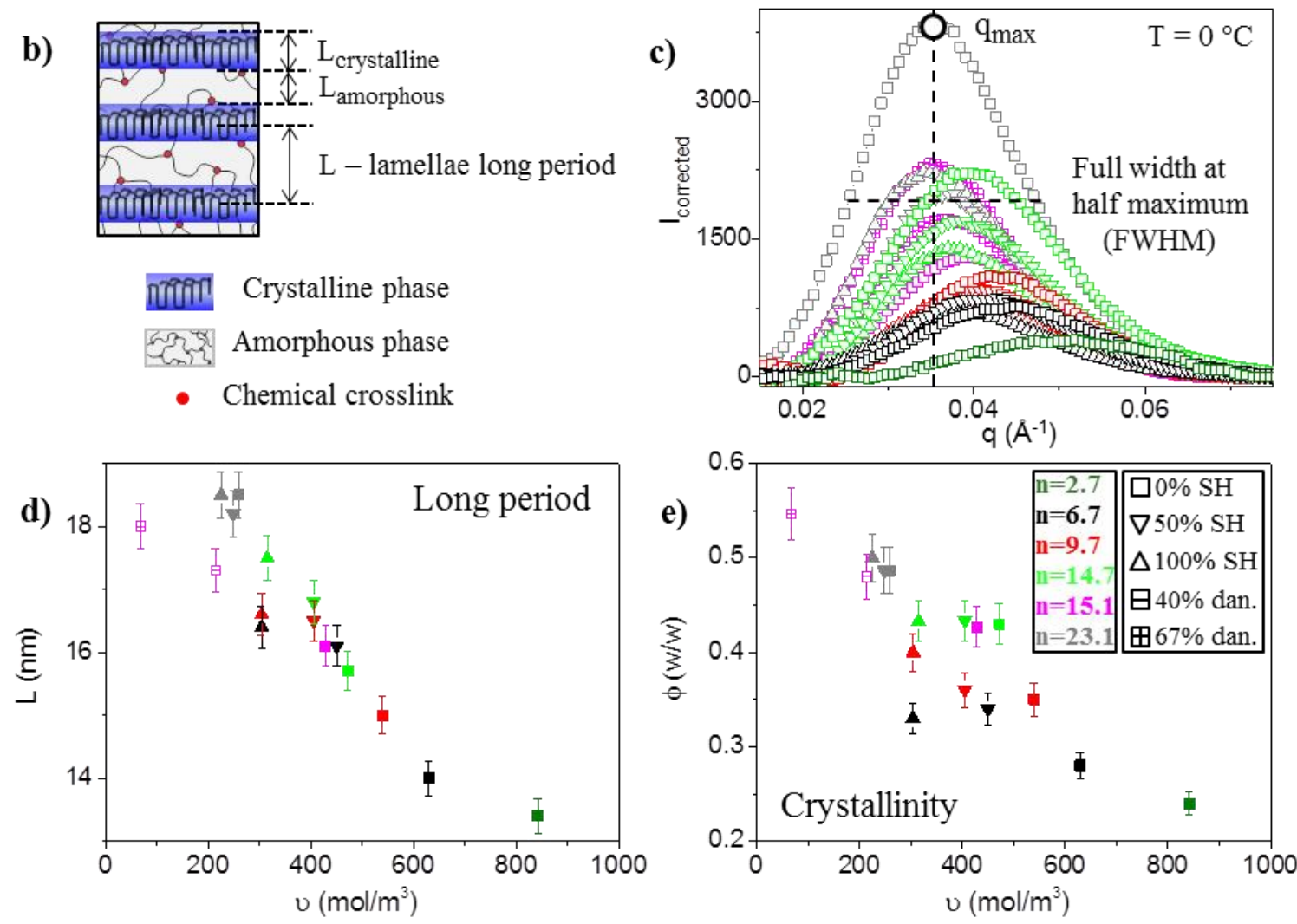

Figure 3. Constrained crystallization: long period and crystallinity. a) AFM height micrographs of spin-coated films of POA networks with three different crosslink densities, determined by degree of oligomerization $n$ as indicated. The micrographs show spherulite branches composed of lamellae stacks (insets). Cross-sectional profiles (along dashed lines in the insets) display an increase of spacing between lamellae with $n$. The spacing was averaged over 100 lines in a chosen area. b) Molecular interpretation of stacks of folded-chain lamellae 
separated by an amorphous phase composed of chain folds, crosslink junctions, uncrystallized strand sections. c) SAXS spectra after subtracting the polynominal decay function-fitted baseline were measured at $0{ }^{\circ} \mathrm{C}$. Peaks were fitted using Lorentz function. Position $q_{\max }$ and peak fullwidth-at-half-maximum (FWHM) were determined. d) Lamellae long period $L=2 \pi / q_{\max }$ and e) crystallinity $\phi=\Delta H / \Delta H_{m}^{0}$ decrease with crosslink density. The crystallinity numbers were obtained by DSC $\left(\Delta H_{m}^{0}=150 \mathrm{~J} / \mathrm{g}\right)$ and calibrated with WAXS as described elsewhere ${ }^{\text {[21] }}$.

Network topology and crosslink density greatly affect organization of the crystalline scaffold in semi-crystalline polymer networks. To study these effects, we used a combination of techniques including Atomic Force Microscopy (AFM), Small and Wide Angle X-ray Scattering (SAXS and WAXS), and Differential Scanning Calorimetry (DSC). AFM height micrographs in Figure 3a show morphology of three samples with different crosslink densities, where we see a characteristic organization of spherulite branches, composed of lamellae stacks (insets). With decreasing crosslink density, both size of the bundles and spacing between the stacked lamellae inside a bundle increase. This observation is attributed to the effect of crosslinking, which exerts both topological and dynamic constraints on network strands hindering the crystal growth.

More quantitative information about the crystalline structure was provided by X-ray scattering and DSC. From SAXS intensity profile in Figure 3c, we determined the long period as $L=2 \pi / q_{\max }$, which includes both crystalline and amorphous fractions (Fig. 3b). We have also calculated the full-width-at-half-maximum (FWHM) as a rough indication of the crystalline structure's dispersity, assuming negligible contribution of crystallite size to peak broadening. ${ }^{[29,}$ ${ }^{30]}$ As shown in Figure 3d, the long period decreases almost linearly with crosslink density, resulting in nearly $6 \mathrm{~nm}$ reduction in $L$ between the highest and lowest crosslink density samples. This is consistent with the corresponding decrease of the melting temperature (Table 1) as predicted by the Gibbs-Thomson equation, assuming that the long period is proportional to 
crystal size. Figure 3e shows that the increase of the crosslink density also results in a significant decrease of crystallinity, which was measured by DSC and then calibrated by WAXS ${ }^{[21]}$ as shown in Supporting Information (Figure S4). As an example, sample 2.7-0\% SH with the highest crosslink density shows the smallest crystal size $(L)$, lowest melting temperature $\left(T_{m}\right)$, and least amount of crystallinity $\left(\Delta H_{m}\right)$. Besides crosslink density, the effect of network topology (controlled by $\mathrm{SH} \%$ ) is also significant. As shown in Figure S5 in Supporting Information, the dispersity of crystal size reduces with increasing SH\%. This is ascribed to a less constrained crystallization of more uniform networks exert.

All structural data are summarized in Table 1, which demonstrates consistent variations of the molecular and morphological characteristics. This provides a good foundation for quantitative studies of correlations between network architecture, crystalline morphology, and reversible shape memory (RSM). Some discrepancy in the long period $(L)$ values determined by AFM and SAXS is ascribed to the surface effect on the crystallization process and 2D AFMimaging of a 3D structure. Although the AFM study is less accurate than SAXS with respect to measurements of crystalline dimensions, it provides visual verification of the lamellae morphology. 

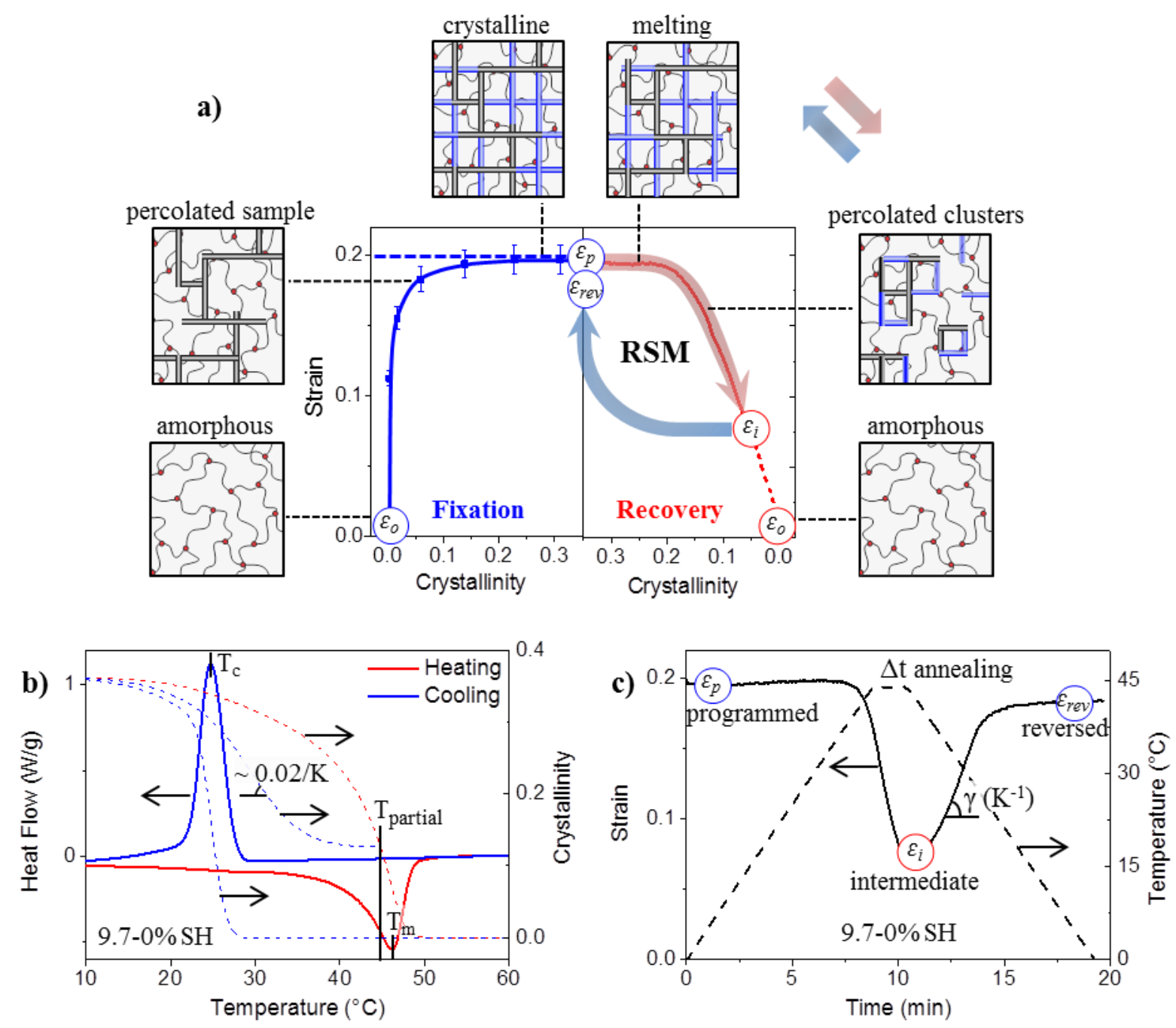

Figure 4. Concept and protocol of reversible shape memory (RSM). a) Shape programming, fixation, and recovery. A programing strain of $\varepsilon_{p}=20 \%$ (dashed blue line) is applied to sample 9.7-0\% SH at $80{ }^{\circ} \mathrm{C}$ and then fixed by quenching to $0{ }^{\circ} \mathrm{C}$. Each data point in the fixation panel corresponds to a strain fixed by quenching at a given stage of the crystallization process. A percolated crystalline scaffold is developed. Crystals in black color indicate early stage (critical) in fixation, blue color indicate the rest 'redundant' crystals in fixation. At final stage of fixation, sample is fixed to the programmed strain $\varepsilon_{p}$. Upon melting, the crystalline scaffold is melted into percolated clusters. At any stage of the melting process, heating can be switched to cooling and reverse the shape transformation from $\varepsilon_{i}$ to $\varepsilon_{r}$. b) A DSC curve of sample 9.7-0\% SH showing melting $T_{m}$ and crystallization transition $T_{c}$, using $5 \mathrm{~K} / \mathrm{min}$ heating/cooling ramp rate. Partial melting temperature $T_{\text {partial }}$ is chosen near $T_{m}$. The integration of melting and crystallization peak 
(enthalpy) divided by the heat of fusion $(\sim 150 \mathrm{~J} / \mathrm{g})$ is calculated as crystallinity, shown in dashed lines. The crystallization rate after cooling from $T_{\text {partial }}$ is about $2 \%$ crystallinity per K (Figure S6 in Supporting Information). c) The strain reversibility is studied as a function of $T_{\text {partial }}$ and annealing time $\Delta t$ at $T_{\text {partial }}$. After programming, a sample with programmed strain $\varepsilon_{p}$ is heated to $T_{\text {partial }}\left(T_{\text {partial }}^{\max }=45^{\circ} \mathrm{C}\right)$. Upon cooling, strain returns to $\varepsilon_{\text {rev }}$. The rate of strain recovery $\gamma\left(\mathrm{K}^{-1}\right)$ is measured as the linear slope multiplying the ramp rate $5 \mathrm{~K} / \mathrm{min}$.

Figure 4a depicts basic steps of reversible shape memory: fixation and recovery. The original (equilibrium) shape is controlled by entropic elasticity of a percolated polymer network secured by chemical crosslinks. To balance the network elasticity and fix a desired strain, a crystalline scaffold is introduced upon cooling (programming step). This scaffold may be viewed as a physical network, which also percolates through the entire sample with a mesh size larger than that of the chemical network. The percolation is essential as it enables fixation of the programmed shape. The left panel of the plot in Figure $4 \mathrm{a}$ demonstrates that less than $5 \%$ crystallinity is required for nearly $100 \%$ shape fixation. This observation is consistent with other shape persistent objects and organisms such as jelly fish supported by ca. $2 \mathrm{wt} \%$ of an organic scaffold [31]. Subsequent crystallization generates an additional substantial fraction of the crystalline phase of ca. $30 \mathrm{wt} \%$. Although these extra crystals are not required for shape fixation, they greatly contribute to the sample rigidity as the Young's modulus increases from the MPa to GPa range during crystallization (Figure S7 in Supporting Information). On heating (right panel of the plot in Figure 4a), the scaffold melts resulting in shape recovery. The shape alteration suggests that the scaffold percolation is interrupted, which presumably yields percolated clusters. The existence of such clusters was indirectly confirmed by measurements and modeling of shear modulus $\left(G \sim \phi^{3}\right)$ of a semi-crystalline polymer network as a function of crystallinity $(\phi)$ during melting ${ }^{[18]}$. On complete melting, the original shape is recovered and stays irreversible upon 
cooling. All samples in this study have high fixation ratio and recovery ratio ranging within $95-$ $99 \%$, which suggests an effective balance between elasticity of the chemical network and rigidity of the crystalline scaffold.

To achieve reversible shape shifting, a programmed sample $\left(\varepsilon_{p}\right)$, is heated to a partial melt state causing partial shape recovery to an intermediate strain $\left(\varepsilon_{i}\right)$. When cooled back, the sample undergoes recrystallization resulting in reverse strain variation towards the programmed shape $\left(\varepsilon_{r e v}\right)$. As discussed previously ${ }^{[18]}$, the reversed shape transformation upon cooling is caused by self-seeding recrystallization of molten chains in the presence of the remaining crystalline scaffold. The extent of reversibility (eq. 3) is controlled by the interplay of two networks: (i) the chemical network, which is partially relaxed at $T=T_{\text {partial }}$ and (ii) the crystalline scaffold, which is partially degraded at $T=T_{\text {partial }}$. While counteracting, both networks provide topological constraints for the molten strands and direct the recrystallization process. If $T_{\text {partial }}$ is too low $\left(T_{\text {partial }}<T_{m}\right)$ and the crystalline fraction is still high, sample is prone to stay at its programmed state without any, or just marginal shape change. If $T_{\text {partial }}$ is too high $\left(T_{\text {partial }}>T_{m}\right)$, the crystalline scaffold is largely destroyed allowing for random pathways for polymer crystallization. By measuring reversibility (eq. 3) as a function of $T_{\text {partial }}$ (Figure S8 in Supporting Information), we determined an optimum temperature $T_{\text {partial }}^{\text {max }}$, which allowed for the greatest reversibility. Table 1 summarizes $T_{\text {partial }}^{\max }$ values determined for each sample and shows that this temperature is near $T_{m}\left(T_{\text {partial }}^{\max } \approx T_{m}\right)$. The counteraction of the chemical and physical networks depends on the crosslink density, which determines both restoring force of the chemical network and recrystallization of the crystalline scaffold. As shown in Figure 5a, reversibility initially increases with crosslink density $(v)$ and then levels off 
at ca. $80 \%$ as it reaches $v>600 \mathrm{~mol} / \mathrm{m}^{3}$. This range of crosslink density corresponds to the irregular networks of Type I (Figure 2b) with shortest strands.
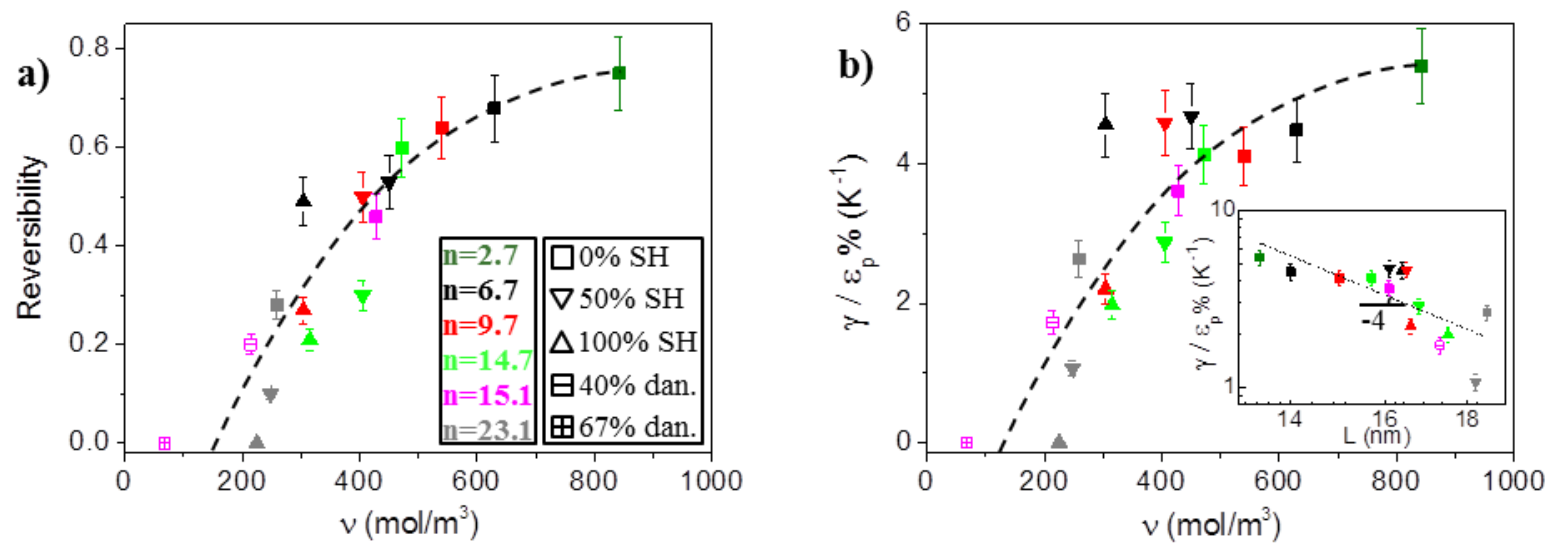

Figure 5. Reversibility and recovery rate: the effect of crosslink density and crystal size. a) Reversibility (eq 3) increases with crosslink density within a range of ca. $50-1000 \mathrm{~mol} / \mathrm{m}^{3}$. Reversibility varies from $0 \%$ to nearly $80 \%$. b) The strain recovery rate $\gamma$ normalized by $\varepsilon_{p}$, increases with crosslink density and decreases with lamellae period $L \sim$ crystal size. A characteristic slope of -4 in the inset has shown great efficiency of smaller crystals.

A similar effect was observed for the recovery rate $\gamma=d \varepsilon / d T$, which is defined in Figure $4 \mathrm{c}$ as strain recovered on $1 \mathrm{~K}$ cooling from the partial melt state. As shown in Figure 5b, sample 2.7-0\% SH with the highest crosslink density $\left(v=842 \mathrm{~mol} / \mathrm{m}^{3}\right)$ recovers $5 \%$ of its programmed strain per $1 \mathrm{~K}$ of temperature decrease, while the looser networks demonstrate progressively slower recovery. In this study, we have applied $5 \mathrm{~K} / \mathrm{min}$ cooling rate. For faster cooling, the recovery process takes only a few seconds, which has been useful the design of a robotic gripper ${ }^{[18]}$. It is also instructive to examine correlation between the recovery rate and crystal size. Using the $L$ vs. $v$ plot in Figure $3 \mathrm{~d}$, we can plot the recovery rate $(\gamma)$ in Figure $5 \mathrm{~b}$ as a function of the lamellae long period $(L)$ (see inset of Figure 5b). The log-log plot shows strong decay of the recovery rate as $\gamma \sim L^{-4}$. A physical interpretation of this scaling relation can be 
developed by expressing the recovery rate as function of crystallinity $\phi \cong n L^{3}$ as $\gamma=\frac{d \varepsilon}{d T}=$ $\frac{d \varepsilon}{d \phi} \frac{d \phi}{d T} \cong \frac{d \varepsilon}{d n} \frac{\dot{\phi}}{L^{3}}$, where $n$ - number of crystallites per unit volume, $\dot{\phi}=d \phi / d T-$ crystallization rate, and $L$ is proportional to the average crystal size. Experimentally, we have measured and shown that the crystallization rate weakly depends on the crosslink density and equal $\dot{\phi}=2 \pm$ $0.1 \mathrm{wt} \% / \mathrm{K}$. By equating the experimentally measured $\gamma \sim L^{-4}$ and the above scaling relation $\gamma \sim \frac{d \varepsilon}{d n} L^{-3}$, we obtain that the amount of strain secured by one crystal increase with decreasing the crystal size as $\frac{d \varepsilon}{d n} \sim L^{-1}$. In other words, materials with smaller crystals allow for higher efficiency of shape fixation.
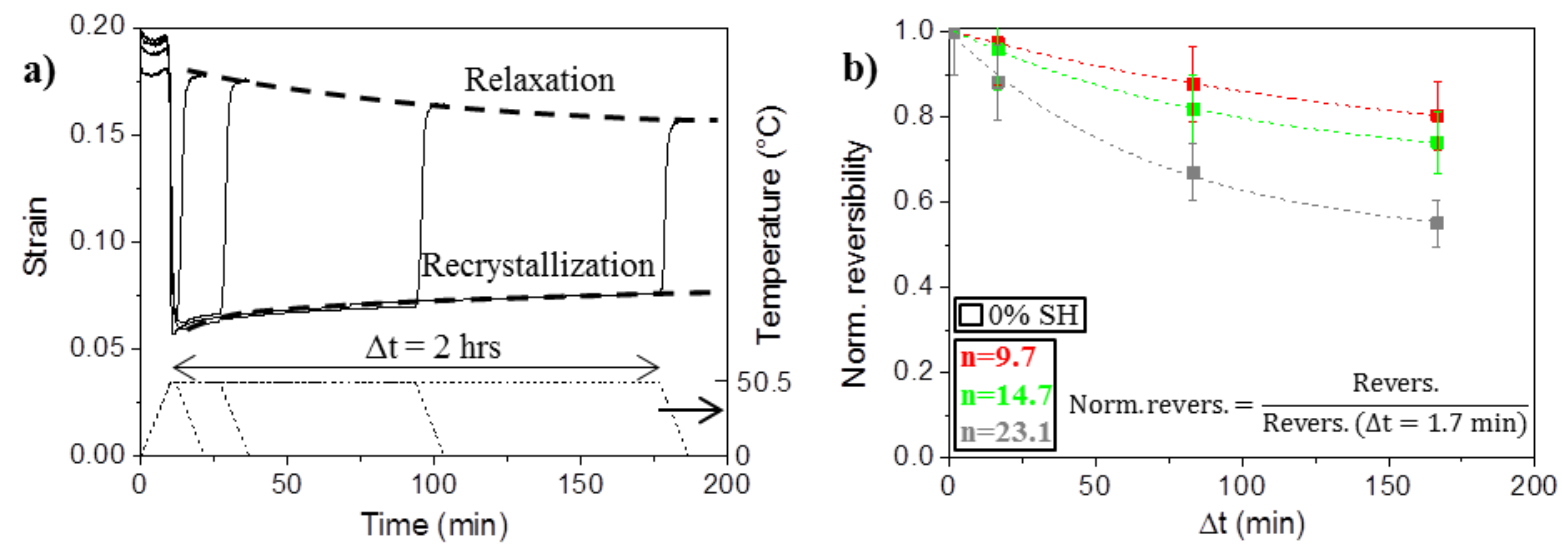

Figure 6. Relaxation and recrystallization. a) Sample $14.7-0 \% \mathrm{SH}$ was used to show the effect of annealing time $\Delta t$ at a partial melt state. A series of temperature ramps $(5 \mathrm{~K} / \mathrm{min})$ was applied including heating to $T_{\text {partial }}^{\max }=50.5^{\circ} \mathrm{C}$, followed by isothermal annealing at $T=50.5^{\circ} \mathrm{C}$ for a controlled duration of $\Delta t$ and cooling to $0{ }^{\circ} \mathrm{C}$. As $\Delta t$ increases, from $1.7 \mathrm{~min}$ to $120 \mathrm{~min}$, the lower dashed line (the strain of partial melt state $\varepsilon_{i}$ ) goes up because of the strain increased by isothermal recrystallization; the upper line (the strain of reversed state $\varepsilon_{\text {rev }}$ ) goes down because of the relaxation of molten chains. Overall, the recoverable portion $\left(\varepsilon_{r e v}-\varepsilon_{i}\right)$ decreases with the annealing time $\Delta t$. b) Fraction of reversible strain (eq 3) decreases with upon relaxation during $\Delta t$ and higher crosslink-density samples show slower decay of reversibility. The Y-axis corresponds 
to normalized reversibility, i.e. fraction of reversible strain (eq 3) at different relaxation times $\Delta t$ is divided by the corresponding fraction at $\Delta t=1.7 \mathrm{~min}$.

The slower shape response of the loose networks is ascribed to faster stress relaxation in the network strands upon melting. To verify this conclusion, we have studied the decay of reversibility during annealing in the partial melt state, when chain relaxation and recrystallization occur simultaneously. While relaxation of molten chains reduces their correlation with the programmed shape leading in a decrease of $\varepsilon_{r e v}$, isothermal recrystallization causes spontaneous increase in the intermediate strain $\varepsilon_{i}$ towards the programmed shape $\varepsilon_{p}$. As such, the reversible strain fraction $\Delta \varepsilon=\varepsilon_{r e v}-\varepsilon_{i}$ decrease from both sides, i.e. due to decreases of $\varepsilon_{r e v}$ and increase of $\varepsilon_{i}$, resulting in the decay of reversibility (eq. 3). However, the decay of reversibility is much lower in the samples with higher crosslink density. For example, 9.7-0\% SH can maintain more than $85 \%$ of its initial reversibility after relaxing for $\Delta t=2$ hours at $T=T_{\text {partial }}^{\max }$. For short time variations (minutes), no measurable decay of reversibility was observed.

\section{CONCLUSTION}

Our understanding of reversible shape memory (RSM) has been advanced through accurate tuning of the architecture of semi-crystalline poly(octylene adipate) networks. Systematic variation of the network-strand length along with molar fractions of dangling chains and tri-thiol cross-linkers provided valuable correlations between the network topology, crosslink density, and mechanical properties (extensibility, modulus) of polymer network in a melt state. These variations of the network architecture directly affect the crystalline scaffold properties (crystallinity and crystal size), as chemical crosslinks exert significant constraints on the 
crystallization process. Even though, the irregular and dense polymer networks generate smaller crystals with broader size distribution, they are more effective for shape control. The RSM properties (degree of reversibility and shape recovery rate) are shown to be mainly controlled by crosslink density, whereas the network topology plays a minor role for RSM. The developed structure-property correlations provide guidelines for network design targeting different RSM applications. For example, dense irregular network are suitable for fabrication of actuators that require small strain and fast cyclic motion, whereas loose networks allow for large-strain, yet, one-time actuation.

\section{ASSOCIATED CONTENT}

\section{Supporting Information}

Characterization of crystallization process (crystallinity, dispersity of crystal size, crystallization rate), strain effect on modulus, and reversibility dependence on partial melting temperature. This material is available free of charge via the Internet at http://pubs.acs.org.

\section{AUTHOR INFORMATION}

\section{Corresponding Author}

*(S.S.S.) E-mail: sergei@email.unc.edu.

\section{Author Contributions}


Q.L. and J.Z. performed the experiments, Q.L. and M.V. synthesized materials, D.N. and O.G. performed SAXS and WAXS measurements, S.S.S. supervised the project and contributed to the revisions of manuscript.

\section{Acknowledgements}

We acknowledge financial support from the National Science Foundation DMR 1122483, DMR1121107, DMR 1407645, and DMR 1436201. This research used resources of the Center for Functional Nanomaterials, which is a U. S. DOE Office of Science Facility, at Brookhaven National Laboratory under Contract No. DE-SC0012704.

\section{REFERENCES}

[1] S. Ashley, Artificial muscles. Scientific American, 2003, 289, 52-59.

[2] B. Mosadegh, A. D. Mazzeo, R. F. Shepherd, S. A. Morin, U. Gupta, I. Z. Sani, D. Lai, S. Takayama and G. M. Whitesides, Control of soft machines using actuators operated by a Braille display. Lab Chip, 2014, 14, 189-199.

[3] D. Liu, D. J. Broer, New insights into photoactivated volume generation boost surface morphing in liquid crystal coatings. Nature, 2015, 6, 8334-1/7.

[4] D. K. Shenoy, D. L. Thomsen, A. Srinivasan, P. Keller, B. R. Ratna, Carbon coated liquid crystal elastomer film for artificial muscle applications. Sens. Actuators, 2002, 96, 184-188.

[5] C. Liu, H. Qin and P. T. Mather, Review of progress in shape-memory polymers. J. Mater. Chem., 2007, 17, 1543-1558.

[6] A. Lendlein and S. Kelch, Shape-Memory Polymers. Angew. Chem. Int. Ed., 2002, 41, 2034-2057.

[7] T. Xie, Recent advances in polymer shape memory. Polymer, 2011, 52, 4985-5000. 
[8] W. Lehmann, H. Skupin, C. Tolksdorf, E. Gebhard, R. Zentel, P. Kruger, M. Losche and F. Kremer, Giant lateral electrostriction in ferroelectric liquid-crystalline elastomers. Nature, 2001, $410,447-450$.

[9] D. L. Thomsen, III, P. Keller, J. Naciri, R. Pink, H. Jeon, D. Shenoy and B. R. Ratna, Liquid crystal elastomers with mechanical properties of a muscle. Macromolecules, 2001, 34, 5868-5875.

[10] D. K. Shenoy, D. L. Thomsen, III, A. Srinivasan, P. Keller and B. R. Ratna, Carbon coated liquid crystal elastomer film for artificial muscle applications. Sens. Actuators, A, 2002, 96, 184-188.

[11] K. Hiraoka, W. Sagano, T. Nose and H. Finkelmann, Biaxial shape memory effect exhibited by monodomain chiral smectic C elastomers. Macromolecules, 2005, 38, 7352-7357.

[12] T. H. Ware, M. E. McConney, J. J. Wie, V. P. Tondiglia and T. J. White, Voxelated liquid crystal elastomers. Science, 2015, 347 (6225), 982-984.

[13] T. Chung, A. Romo-Uribe and P. T. Mather, Two-Way Reversible Shape Memory in a Semicrystalline Network. Macromolecules, 2008, 41, 184-192.

[14] J. Li, W. R. Rodgers and T.Xie, Semi-crystalline two-way shape memory elastomer. Polymer, 2011, 52, 5320-5325.

[15] S. Pandini, S. Passera, M. Messori, K. Paderni, M. Toselli, A. Gianoncelli, E. Bontempi and T. Ricco, One-way and two-way shape memory behaviour of semi-crystalline networks based on sol-gel cross-linked poly(e-caprolactone). Polymer, 2012, 53, 1915-1924.

[16] K. K. Westbrook, P. T. Mather, V. Parakh, M. L. Dunn, Q. Ge, B. M. Lee, and H. J. Qi, Two-way reversible shape memory effects in a free-standing polymer composite. J. Smart Materi. Struct., 2011, 20, 065010/1-9.

[17] M. Behl, K. Kratz, U. Noechel, T. Sauter, and A. Lendlein, Temperature-memory polymer actuators. PNAS, 2013, 110, 12555-12559.

[18] J. Zhou, S. A. Turner, S. M. Brosnan, Q. Li, J. Y. Carrillo, D. Nykypanchuk, O. Gang, V. S. Ashby, A. V. Dobrynin and S. S. Sheiko, Shapeshifting: Reversible Shape Memory in Semicrystalline Elastomers. Macromolecules, 2014, 47, 1768-1776. 
[19] S. A. Turner, J. Zhou, S. S. Sheiko, V. S. Ashby, Switchable Micropatterned Surface Topographies Mediated by Reversible Shape Memory. ACS Appl. Mater. Interfaces, 2014, 6, 8017-8021.

[20] C. Tippets, Q. Li, Y. L. Fu, E. Donev, J. Zhou, S. A. Turner, A-M. Jackson, V. S. Ashby, S. S. Sheiko and R. Lopez, Dynamic Optical Gratings Accessed by Reversible Shape Memory. ACS Applied Materials \& Interfaces, 2015, 7, 14288-14293.

[21] J. Zhou, Q. Li, S. A. Turner, V. S.s Ashby and S. S. Sheiko, Isothermal programming of triple shape memory. Polymer, 2015, 72, 464-470.

[22] H. R. Allcock, F. W. Lampe and J. E. Mark, Contemporary Polymer Chemistry (3rd ed., Pearson), 2003, 324-330.

[23 ] P. J. Flory, R. R. Garrett, S. Newman, and L. Mandelkern, Thermodynamics of Crystallization in High Polymers. Cellulose Trinitrate. Journal of Polymer Science, 1954, 12, 97-107.

[24] C. E. Hoyle, C. N. Bowman, Thiol-ene Click Chemistry. Angew. Chem. Int. Ed., 2010, 49, 1540-1573.

[25] C. L. Lewis, Y. Meng, and M. Anthamatten, Well-Defined Shape-Memory Networks with High Elastic Energy Capacity. Macromolecules, 2015, 48(14), 4918-4926.

[26] M. Rubinstein and R. H. Colby, Polymer Physics, OUP Oxford, 2003, 264-268.

[27] L. R. G. Treloar, The Physics of Rubber Elasticity. OUP Oxford, 2005, 74-75.

[28] S. K. Patel, S. Malone, C. Cohen, J. R. Gillmor and R. H. Colby, Elastic modulus and equilibrium swelling of poly(dimethylsiloxane) networks. Macromolecules, 1992, 25, $5241-5251$.

[29] C. S. Tsao and H. L. Chen, Concurrent Transformation of Copolymer Domain Morphology Induced by the Order-Disorder Transition of Comb Block in Supramolecular Comb-Coil Block Copolymer, Macromolecules, 2004, 37, 8984-8991.

[30] J. S. C. Loo, C. P. Ooi, F. Y. C. Boey, Degradation of poly(lactide-co-glycolide) (PLGA) and poly(1-lactide) (PLLA) by electron beam radiation, Biomaterials, 2005, 26, 1359-1367. 
[31] A. G. Lowndes, Percentage of water in jelly-fish. Nature, 1942, 150, 2234-235. 